\title{
Measurement of Transverse Single-Spin Asymmetry and Cross-Section Ratios of Kinematically Fully Reconstructed Weak Bosons in p-p Collisions at $\sqrt{s}=500-510 \mathrm{GeV}$ at RHIC
}

\author{
Salvatore Fazio*i \\ Brookhaven National Laboratory \\ E-mail: sfazio@bnl.gov
}

\begin{abstract}
We present the status and preliminary results of the analysis of kinematically fully reconstructed weak bosons at the STAR experiment. The transverse single-spin asymmetry $\left(A_{N}\right)$ has been measured in transversely polarized proton-proton collisions at $\sqrt{s}=500 \mathrm{GeV}$, with a recorded integrated luminosity of $25 \mathrm{pb}^{-1}$. The measured observable is sensitive to the non-universality of the Sivers function, a fundamental prediction from the gauge invariance of $\mathrm{QCD}$, and can provide a direct verification of transverse momentum dependent (TMD) distribution factorization. Furthermore, it provides an ideal tool to study the spin-flavor structure of valence and sea quarks inside the proton and to test the TMD evolution. The $W^{+} / W^{-}$cross section ratio has been measured in unpolarized proton-proton collisions at $\sqrt{s}=500$ and $510 \mathrm{GeV}$, with a recorded integrated luminosity of $110 \mathrm{pb}^{-1}$. The observable is sensitive to the flavor asymmetry of sea quarks, providing an independent constraint on the large flavor asymmetry observed in Drell-Yan experiments, without the assumption of charge symmetry required in fitting the Drell-Yan data.
\end{abstract}

XXIII International Workshop on Deep-Inelastic Scattering,

27 April - May 12015

Dallas, Texas

* Speaker.

${ }^{\dagger}$ For the STAR Collaboration 


\section{Introduction}

Understanding the partonic structure of the proton in multi-dimensions has become a hot topic in the past decade [1]. Transversely polarized spin effects can be used to access transverse momentum dependent (TMD) [2] parton distribution functions (PDFs), which contain information on the intrinsic transverse momentum of a parton, together with the fraction that the same parton carries of the longitudinal momentum of the parent nucleon, leading to a 2+1 dimensional picture of the proton. Drell-Yan di-lepton (DY) and $W^{ \pm} / Z^{0}$ boson production in p-p collisions are observables which provide the two scales typically required to apply the TMD framework to transverse single-spin asymmetries, one hard scale is given by the invariant mass, while a soft scale is given by the transverse momentum. A particularly interesting TMD is the so-called Sivers function [3], $f_{1 T}^{\perp}$, which describes the correlation of parton transverse momentum with the transverse spin of the nucleon.

There is evidence of a quark Sivers effect in semi-inclusive DIS (SIDIS) measurements [4] where the quark Sivers function is associated with a final state effect from the gluon exchange between the struck quark and the target nucleon remnants. On the other hand, for the $D Y$ process or the $W^{ \pm} / Z^{0}$ production in p-p collisions, the Sivers asymmetry originates from the initial state of the interaction. As a consequence, the quark Sivers functions are of opposite sign in SIDIS and in $D Y / W^{ \pm} / Z^{0}[5]$

$$
f_{q / h^{\uparrow}}^{S I D I S}\left(x, k_{\perp}\right)=-f_{q / h^{\uparrow}}^{D Y / W^{ \pm} / Z^{0}}\left(x, k_{\perp}\right),
$$

and this non-universality is a fundamental prediction from the gauge invariance of QCD.

The experimental test of this sign change is one of the open questions in hadronic physics, and can provide insights on the TMD factorization. While luminosity and experimental requirements for a meaningful measurement of asymmetries in Drell-Yan production are challenging, weak boson production is also sensitive [6] to the predicted sign change and can be measured at STAR.

Thanks to the high $Q^{2} \simeq M_{W^{ \pm}}^{2} / Z^{0}$ scale, the weak boson production provides also a stringent test of the TMD evolution [6]. Furthermore, the $W^{+}\left(W^{-}\right)$boson, which is produced through $u+\bar{d}(d+\bar{u})$ annihilation, can provide essential input to disentangle the contribution to the Sivers function of light-sea quarks, which is essentially unconstrained by fits to SIDIS data [6]. The STAR experiment at RHIC is currently the only place in the world where all these effects can be tested simultaniously.

The transverse single-spin asymmetry, $A_{N}$, solely calculated from the lepton decay is a very strong function of the lepton kinematics [7] and therefore its measurement is experimentally challenging, thus a full reconstruction of the produced boson kinematics is crucial for a meaningful measurement. Based on the transversely polarized data sample corresponding to a luminosity of $L=25 \mathrm{pb}^{-1}$ collected in the year 2011 at $\sqrt{s}=500 \mathrm{GeV}$, an analysis has been performed at STAR to fully reconstruct the $W^{ \pm}$bosons from the lepton decay and all other particles in the recoil from the initial hard scattering. The preliminary results have been presented for the first time at the DIS 2014 workshop [8]. This analysis also includes a first look at $A_{N}$ in $Z^{0}$ production. A STAR measurement with higher collected luminosity $\left(L=400 \mathrm{pb}^{-1}\right)$, planned for 2017 , will be directly competitive with a Drell-Yan measurement in pion-proton scattering at CERN [9].

In addition to the study of proton spin phenomena, $W$ boson production can also be used to constrain PDFs for the sea quarks. The E866 experiment, through observing the DY process, 
first measured the $\bar{d} / \bar{u}$ ratio [10] versus Bjorken-x, showing a transition from a dominant $\bar{d}$ to a dominant $\bar{u}$ quark. In the case of weak boson production, the $W^{+}$boson is sensitive to the $\bar{d}$ quark, while the $W^{-}$boson is sensitive to the $\bar{u}$ quark and the information on the sea quark distribution can be extracted from the decomposition of the $W$ cross section charge-ratio

$$
R_{W}=W^{+} / W^{-}=\frac{u\left(x_{1}\right) \bar{d}\left(x_{2}\right)+\bar{d}\left(x_{1}\right) u\left(x_{2}\right)}{\bar{u}\left(x_{1}\right) d\left(x_{2}\right)+d\left(x_{1}\right) \bar{u}\left(x_{2}\right)} .
$$

In this paper we present the STAR preliminary measurement of the $R_{W}=W^{+} / W^{-}$unpolarized cross section ratio in the near valence region, $x>0.1$, using the data collected in the year 2011 (2012) at $\sqrt{s}=500(510) \mathrm{GeV}$, corresponding to a total collected luminosity of $L=102 \mathrm{pb}^{-1}$. More information on the physics motivations and experimental details of the $R_{W}$ measurement are discussed in [11]. This measurement is complementary to LHC, which can measure $R_{W}$ below the valence region: $10^{-3}<x<10^{-1}$.

\section{Data selection and results}

Data were recorded using a calorimeter trigger requirement of $12 \mathrm{GeV}$ of transverse energy $E_{T}$ in a $\Delta \eta \times \Delta \phi$ region of $\sim 0.1 \times 0.1$ of the BEMC. We define a $P_{T}$-balance variable, $\vec{P}_{T}^{b a l}$, as the vector sum of the decay electron $\vec{P}_{T}^{e}$ and the transverse momentum of the hadronic recoil

$$
\vec{P}_{T}^{b a l}=\vec{P}_{T}^{e}+\vec{P}_{T}^{\text {recoil }}
$$

where $\vec{P}_{T}^{\text {recoil }}$ is the vector sum of the transverse momentum of all the tracks not belonging to the decay electron candidate and all the trackless clusters in the BEMC with an energy above the noise threshold of $200 \mathrm{MeV}$.

As done in the STAR previous analyses of weak boson longitudinal spin asymmetry [12] and cross sections [13], a data sample characterized by the $W \rightarrow e v$ signature has been selected requiring an isolated high $P_{T}>25 \mathrm{GeV}$ electron. All tracks must come from a single vertex with $\left|Z_{\text {vertex }}\right|<100 \mathrm{~cm}$. In order to reject QCD background events the scalar variable signed- $P_{T^{-}}$ balance $=\left(\vec{P}_{T}^{b a l} \cdot \vec{P}_{T}^{e}\right) /\left|\vec{P}_{T}^{e}\right|$ is required to be larger than $18 \mathrm{GeV}$. The charge misidentification between the $W^{+}$and the $W^{-}$samples is minimized by requiring $0.4<\mid\left(\right.$ Charge $\left.\times E_{T}^{e}\right) / P_{T}^{e} \mid<1.8$.

In reconstructing the $W$ boson kinematics, the momentum of the neutrino produced in the leptonically decayed $W$ can only be indirectly deduced from conservation of the transverse momentum: $\vec{P}_{T}^{W}=-\vec{P}_{T}^{\text {recoil }}$.

At the STAR detector, due to a limited acceptance of $|\eta|<1$, the challenge with measuring the missing momentum from the hadronic recoil is that particles at high pseudo-rapidities are not detected. At the same time, those recoil particles carry away only a little portion of the total transverse momentum. We accounted for the unmeasured tracks and clusters by using an event-by-event Monte Carlo correction to the data (we used PYTHIA 6.4 [14] with "Perugia 0" tune [15]). The correction factor to the measured $W$ transverse momentum in the $i$-th bin is defined as $k_{i}=P_{T, i}^{W}$ (true) $/ P_{T, i}^{\text {recoil }}$ (reconstructed), where $P_{T, i}^{W}$ (true) is the $P_{T}$ of the $W$ generated by the Monte Carlo and $P_{T, i}^{\text {recoil }}$ (reconstructed) is the $P_{T}$ of the recoil reconstructed in each $i$-th bin after a full simulation of the detector and applying all the selection requirements. The measured 
value of the boson $P_{T}$ in each event was then corrected by randomly sampling a value from the corresponding $P_{T}$-bin of the normalized correction factor distribution.

In reconstructing the hadronic recoil from the tracks and clusters, we rejected events with a total $P_{T}^{\text {recoil }}<0.5 \mathrm{GeV}$, a region where the correction factor average value becomes high and its distribution very wide. We also required each single track in the recoil to have a $P_{T}>0.2 \mathrm{GeV}$.

Knowing its transverse momentum, the longitudinal component of the neutrino's momentum can be reconstructed solving the quadratic equation for the invariant mass of the produced boson

$$
M_{W}^{2}=\left(E_{e}+E_{v}\right)^{2}-\left(\vec{P}_{e}+\vec{P}_{v}\right)^{2},
$$

where we assumed the nominal value of the $W$-mass. Eq. 2.2 leads to two possible solutions for $P_{L}^{V}$, and we chose the smaller one in magnitude which, as shown by a Monte Carlo study, gives a more accurate reconstruction of the original kinematics.

Background contribution coming from $W^{ \pm} \rightarrow \tau^{ \pm} v_{\tau}, Z^{0} \rightarrow e^{+} e^{-}$has been studied using PYTHIA 6.4, whereas background from QCD events has been studied using a data driven procedure reversing our signed- $P_{T}$-balance selection cut. All background sources have been estimated to be at most a few percent of the selected sample.

The transverse single-spin asymmetry is expressed as: $A_{N}=\frac{\sigma_{\uparrow}-\sigma_{\downarrow}}{\sigma_{\uparrow}+\sigma_{\downarrow}}$, where $\sigma_{\uparrow \downarrow}$ is the cross section measured with an up/down polarization of the proton beam. The data sample is binned in three observables: the rapidity $y^{W}$, the $P_{T}^{W}$, and the azimuthal angle $\phi$ of the produced boson. Thus, we calculate the transverse single-spin asymmetry using the following formula, which cancels out false asymmetries due to geometry and luminosity [16]

$$
A_{N}^{\sin (\phi)}=\frac{1}{\langle P\rangle} \frac{\sqrt{N_{\uparrow}\left(\phi_{i}\right) N_{\downarrow}\left(\phi_{i}+\pi\right)}-\sqrt{N_{\uparrow}\left(\phi_{i}+\pi\right) N_{\downarrow}\left(\phi_{i}\right)}}{\sqrt{N_{\uparrow}\left(\phi_{i}\right) N_{\downarrow}\left(\phi_{i}+\pi\right)}+\sqrt{N_{\uparrow}\left(\phi_{i}+\pi\right) N_{\downarrow}\left(\phi_{i}\right)}}
$$

where $N$ is the number of reconstructed events to the "left" $(\phi)$ or to the "right" $(\phi+\pi)$ side respectively to the nominal polarization vector in collisions with an up/down $(\uparrow \downarrow)$ beam helicity configuration, with an average RHIC beam polarization for 2011 transverse proton-proton collisions of $\langle P\rangle=(53 \pm 3.4) \%$. The $A_{N}$ amplitude has been extracted performing a $\sin (\phi)$ fit in each of the $y^{W}-P_{T}^{W}$ kinematic bins.

The STAR preliminary results for the $A_{N}$ measurement of the $W^{+}$and $W^{-}$boson production are shown separately in Fig. 1 as a function of $y^{W}$ and $P_{T}^{W}$. The systematic uncertainties, added in quadrature, have been evaluated via a Monte Carlo test using a theoretical prediction for the asymmetry from [6]. The $3.4 \%$ overall systematic uncertainty on beam polarization measurement is not shown in the plots.

The transverse single-spin asymmetry of the $Z^{0} \rightarrow e^{+} e^{-}$process has been also measured because it has many advantages: it is experimentally very clean and the boson kinematics are easily reconstructed from the two decay leptons produced at central rapidities, well within the acceptance of the STAR detector. Thus, the measurement is background free, carries only the overall systematic uncertainty coming from the polarization measurement, and the asymmetry is expected to be of the same size as that of the $W^{ \pm}$. The only challenge comes from the much lower cross section of the $Z^{0} \rightarrow e^{+} e^{-}$process, which requires the collection of a very large data sample for a statistically significant measurement. 

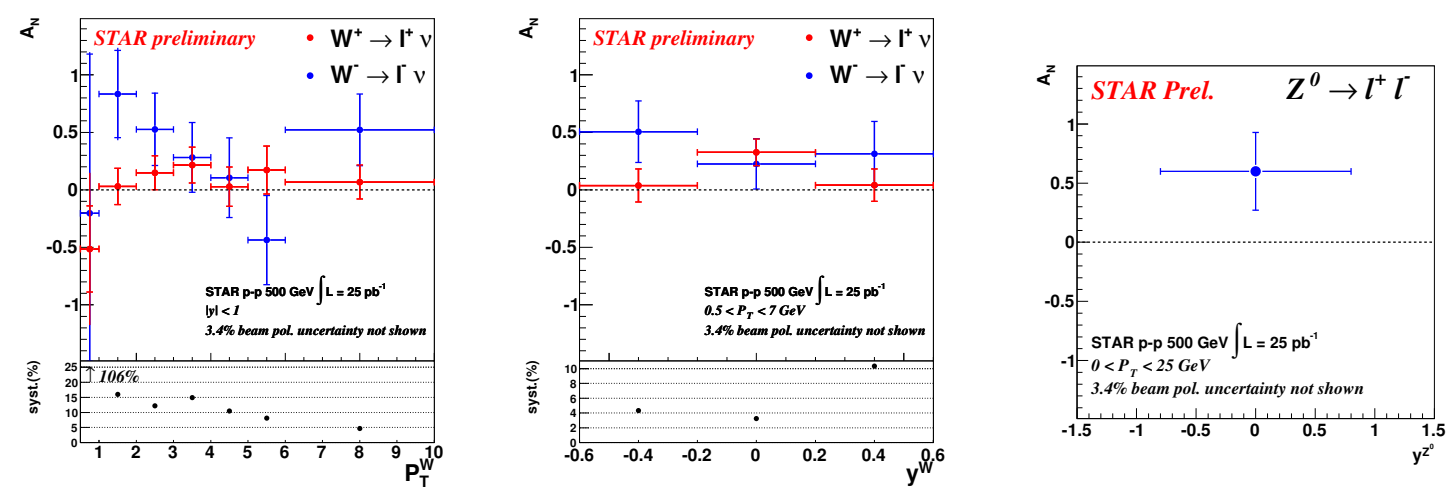

Figure 1: Transverse single-spin asymmetry amplitude for $W^{ \pm}$and $Z^{0}$ boson production measured at STAR in a pilot run at $\sqrt{s}=500 \mathrm{GeV}$ with a recorded luminosity of $25 \mathrm{pb}^{-1}$.

A data sample characterized by the $Z^{0}$ signature has been selected, requiring two high $P_{T}>$ $25 \mathrm{GeV}$ electrons, of opposite charge and with an invariant mass within $\pm 20 \%$ of the nominal value. The STAR preliminary result for the $A_{N}$ measurement of the $Z^{0}$ boson production in a single $y^{Z}, P_{T}^{Z}$ bin is shown in Fig. 1 .

The unpolarized cross section ratio has been measured as

$$
R_{W}=\frac{\sigma\left(W^{+}\right)}{\sigma\left(W^{-}\right)}=\frac{N_{o b s}^{W^{+}}-N_{b k g}^{W^{+}}}{N_{o b s}^{W^{-}}-N_{b k g}^{W^{-}}} \cdot \frac{\varepsilon^{W^{-}}}{\varepsilon^{W^{+}}},
$$

where $N_{o b s}^{W^{ \pm}}$corresponds to the number of observed $W^{ \pm}$bosons, $N_{b k g}^{W^{ \pm}}$is the number of background events in each sample and $\varepsilon^{W^{ \pm}}$is the efficiency of selecting a $W^{ \pm}$event. The charge dependance of the efficiency has been found to be minimal, therefore the efficiency factors give a negligible contribution to the $R_{W}$ measurement.

The preliminary measurement of the $R_{W}$ versus the decay electron pseudo-rapidity and the produced $W$ boson rapidity are shown in Fig. 2 and Fig. 3 respectively and compared to different theoretical predictions.

\section{Conclusions and outlook}

Our study demonstrates the capability of STAR to perform measurements with fully reconstructed $W^{ \pm}$bosons. The preliminary results for the $A_{N}$ from Fig. 1 can be compared with the most up-to-date theoretical $A_{N}$ predictions for $W^{ \pm}, Z^{0}$ boson production including TMD-evolution from reference [6], shown in Fig. 4, where the error bands have been updated accounting for the current almost complete uncertainty on sea-quark functions in the fits [17]. RHIC plans to collect $400 \mathrm{pb}^{-1}$ events of transversely polarized p-p collisions at $\sqrt{s}=510 \mathrm{GeV}$ during the 2017 run, using a dynamic $\beta^{*}$ squeeze [18] throughout the fill. This will allow for a precise $A_{N}$ measurement of weak boson production at STAR (for projections see [8]), and can lead to the first experimental test of the sign change of the Sivers function if the evolution proves to be smaller than a factor $\sim 5$. Furthermore it will provide an ideal tool to study the spin-flavor structure of sea quarks inside the proton. 


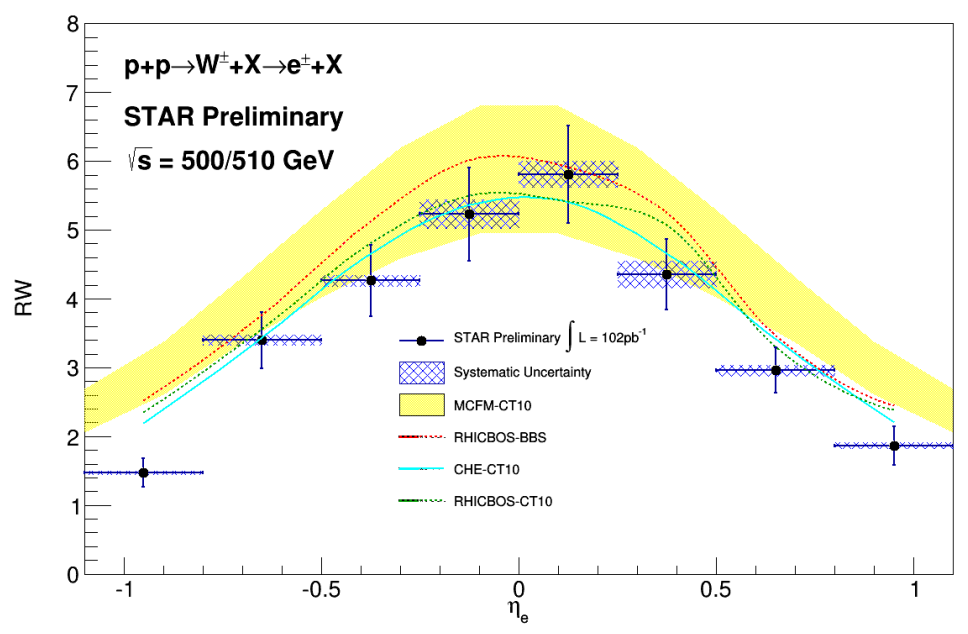

Figure 2: $R_{W}=W^{+} / W^{-}$as a function of the decay electron pseudo-rapidity. Vertical bars on the data points represent the statistical uncertainty only.

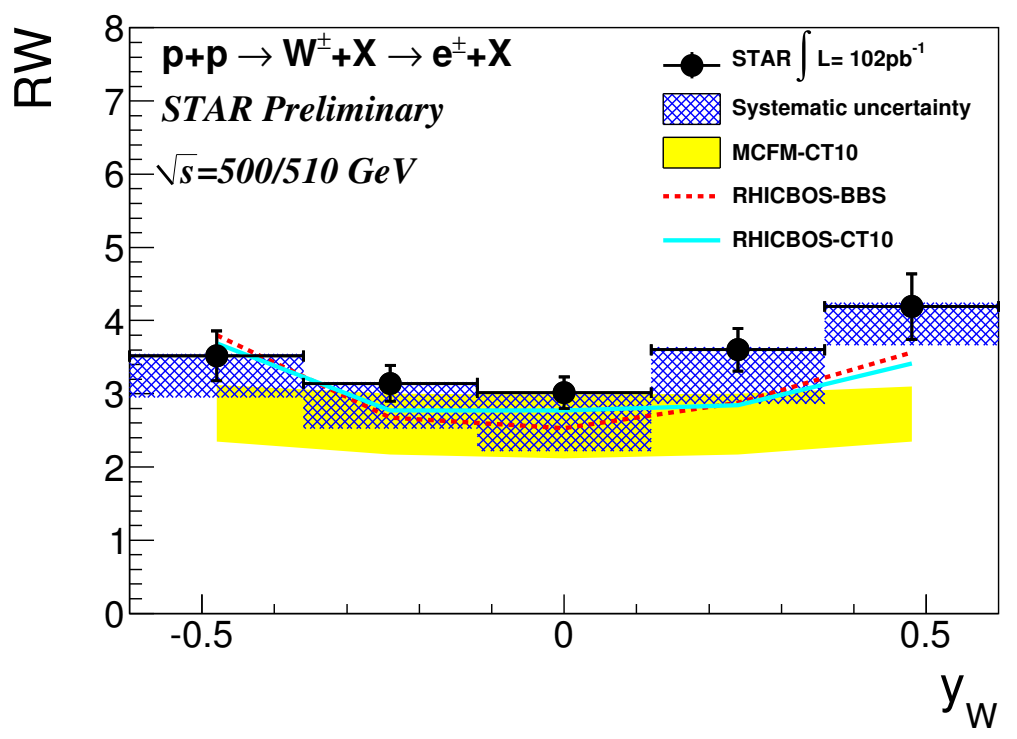

Figure 3: $R_{W}=W^{+} / W^{-}$as a function of the $W$ boson rapidity. Vertical bars on the data points represent the statistical uncertainty only.

We have also obtained a preliminary measurement of the unpolarized cross section chargeratio, $R_{W}$, versus both the decay electron pseudo-rapidity and the produced $W$ boson rapidity combining the data samples from the 2011 and the 2012 RHIC runs, corresponding to a collected luminosity of $102 \mathrm{pb}^{-1}$. Once the calibration of the already collected data from the 2013 RHIC run $\left(L=250 \mathrm{pb}^{-1}\right)$ is completed, the statistical precision of the measurement will be further improved. Including these results in global PDF fits will help to constrain sea quark distribution in the near valence region $(x>0.1)$. 

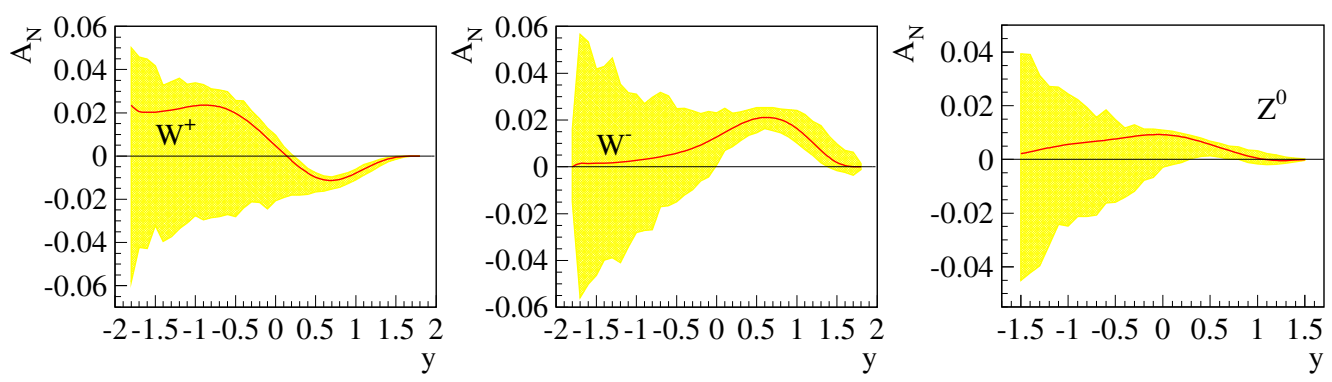

Figure 4: Theoretical prediction of $A_{N}$ for $W^{ \pm}$and $Z^{0}$ boson production in $\mathrm{p}+\mathrm{p}$ collisions at $\sqrt{s}=500 \mathrm{GeV}$ including TMD-evolution [6].

\section{References}

[1] Kai-bao Chen, Shu-yi Wei, Zuo-tang Liang, arXiv:1506.07302 [hep-ph]

[2] S. Mert Aybat, and Ted C. Rogers, Phys. Rev. D 83, 114042 (2011)

[3] D. W. Sivers, Phys. Rev. D41, 83 (1990), 43, 261 (1991)

[4] M. Anselmino et al., Eur. Phys. J. A39, 89 (2009)

[5] J. C. Collins, Phys. Lett. B536, 43 (2002)

[6] M. G. Echevarria, A. Idilbi, Z.-B. Kang, I. Vitev, Phys. Rev. D89, 074013 (2014)

[7] Z.-B. Kang and J. -W. Qiu, Phys. Rev. Lett. 103, 172001 (2009)

[8] S. Fazio and D. Smirnov (for the STAR Collaboration), PoS (DIS2014) 237

[9] C. Quintans, PoS(DIS2014)240

[10] E. A. Hawker et al., Phys. Rev. Lett. 80, 3715 (1998)

[11] M. Posik (for the STAR Collaboration), these Proceedings

[12] L. Adamczyk et al., The STAR Collaboration, Phys. Rev. Lett. 113, 072301 (2014)

M. M. Aggarwal et al., The STAR Collaboration, Phys. Rev. Lett. 106, 062002 (2011)

[13] G. Agakishiev et al., The STAR Collaboration, Phys. Rev. D 85, 092010 (2012)

[14] https://pythia6.hepforge.org/

[15] Peter Z. Skands, Phys. Rev. D82, 074018 (2010)

[16] Bültmann S et al., Phys. Lett. B632 167 (2006)

Bültmann S et al., Phys. Lett. B647 98 (2007)

Ohlsen G G and Keaton Jr P W, Nucl. Instr. Meth. 10941 (1973)

[17] Z.-B. Kang, private communication

[18] D. Trbojevic, J. Yichao, Y. Luo, BNL-102458-2013-CP 